\title{
ABSOLUTELY FG SPACES
}

\author{
A. H. STONE
}

\begin{abstract}
This paper gives characterizations of (a) the Hausdorff spaces that are the intersection of an open set and a closed set in every Hausdorff space containing them, (b) the spaces all of whose subspaces have the property in (a). The last extends a theorem of Herrlich, Kannan and Rajagopalan on hereditarily locally compact spaces.
\end{abstract}

0. Introduction. Recently, Herrlich, Kannan and Rajagopalan [2] have characterized the (Hausdorff) spaces that are hereditarily locally compact. It is well known that the locally compact spaces are closely related to the absolutely FG spaces - the spaces that in every imbedding are the intersection of a closed set and an open set. ${ }^{1}$ Thus it is of interest to study the Hausdorff spaces that are hereditarily absolutely FG. We shall see that they coincide with the hereditarily locally compact spaces, thereby sharpening the theorem of [2], and incidentally obtaining a somewhat simpler description of these spaces than the one in [2].

1. Absolutely FG spaces. A subset $E$ of a topological space $(X, \mathcal{T})$ will be said to be "FG" providing it is the intersection of a closed set and an open set. As is well known [1, I.3.3, Proposition 5] this is equivalent to saying that $E$ is the difference between two open sets, or between two closed ones, or that $E$ is locally closed, or (the criterion usually most convenient) $E$ is open relative to its closure $\bar{E}$. We shall say that a space $(X, \mathcal{T})$ is $A(\mathrm{FG})$ providing $X$ is $\mathrm{FG}$ in every topological space $Y$ of which $(X, \mathcal{T})$ is a subspace. More generally, if $\mathrm{P}$ is a property, we say $(X, \mathcal{T})$ is $A(\mathrm{FG})(\mathrm{P})$ providing $(X, \mathcal{T})$ has $\mathrm{P}$ and $X$ is $\mathrm{FG}$ in every space having $\mathrm{P}$ of which $(X, \mathcal{T})$ is a subspace. We are mainly concerned with the cases in which $P$ is a separation axiom $\left(\mathrm{T}_{0}-\mathrm{T}_{4}\right)$ or metrizability (abbreviated to Metr.). The desirability of properties stronger than the $T_{1}$ axiom is shown by the first two theorems below; we shall accordingly concentrate on $T_{2}$ spaces. However, all separation axioms assumed will be mentioned explicitly.

THEOREM 1. For an arbitrary space $X$, the following are equivalent: (1) $X$ is $A(F G),(2) X$ is $A(F G)\left(\mathrm{T}_{0}\right),(3) X=\varnothing$.

For if $X \neq \varnothing$, pick $a \in X$ and fix an infinite set $Z$ disjoint from $X$. Let $\mathcal{C}$ be the

Received by the editors January 11, 1980.

1980 Mathematics Subject Classification. Primary 54C25, 54B05, 54D45; Secondary 54B17, 54D35.

Key words and phrases. Absolutely closed, locally absolutely closed, absolutely locally closed, hereditarily locally compact, resolvable, maximally irresolvable.

${ }^{1}$ In fact, every locally compact space is FG in every Hausdorff space containing it, while conversely, if $X$ is Tychonoff and is FG in $\beta X$, then $X$ is locally compact. 
family of cofinite subsets of $Z$. Put $Y=X \cup Z$, and define a topology on $Y$ by specifying open neighborhood bases $\mathcal{Q}(y)$ at each $y \in Y$ as follows.

(i) If $y \in \bar{a}$, the closure of $\{a\}$ in the given space $(X, \mathcal{T}), \mathcal{Q}(y)$ consists of all sets $U \cup C$ where $y \in U \in \mathcal{T}$ and $C \in \mathcal{C}$.

(ii) If $y \in X \backslash \bar{a}$, $Q(y)$ consists of the sets $U$ such that $y \in U \in \mathcal{T}$ and $a \notin U$.

(iii) If $y \in Z, \mathcal{Q}(y)$ consists of the sets $U \cup C$ where $a \in U \in \mathcal{T}$ and $C \in \mathcal{C}$.

It is easy to verify that this does define a topology $\mathcal{T}^{*}$ on $Y$, that $(X, \mathcal{T})$ is a subspace of $\left(Y, \mathcal{T}^{*}\right)$, and that $\left(Y, \mathcal{T}^{*}\right)$ is $\mathrm{T}_{0}$ if $(X, \mathcal{T})$ is $\mathrm{T}_{0}$. Further, $X$ is dense in $Y$ but not open (since every neighborhood of $a$ meets $Z$ ). Thus $X$ is not FG in $Y$.

REMARK. A simplified version of this construction (with $Z$ replaced by a singleton and $\mathcal{C}$ by $\{Z\}$ ) will similarly show that the empty set is the only $A(\mathrm{FG})$ (finite) space.

\section{Theorem 2. $X$ is $A(F G)\left(\mathrm{T}_{1}\right)$ if and only if $X$ is discrete.}

Suppose $X$ is a $T_{1}$ space, with topology $\mathcal{T}=\left\{G_{\alpha}: \alpha \in A\right\}$, indexed in a one-toone way by a set $A$ disjoint from $X$. Put $Y=X \cup A$, with topology $\sigma^{*}$ having the collection of all sets

$$
\left(G_{\alpha} \cup\left\{\beta: G_{\beta} \subset G_{\alpha}\right\}\right) \backslash F,
$$

where $\alpha, \beta \in A$ and $F$ is finite, as a basis. One verifies without difficulty that this does define a $T_{1}$ topology, and that $(X, \mathcal{T})$ is a subspace of $\left(Y, \mathcal{T}^{*}\right)$. Further, the closure $\bar{X}$ of $X$ in $\left(Y, \mathcal{J}^{*}\right)$ is $X \cup\left\{\alpha: G_{\alpha}\right.$ is infinite $\}$. Thus if $X$ is FG in $Y$, it is open in $\bar{X}$, and that implies that $X$ is discrete, for the following reason. If $X$ is not discrete, take $x_{0} \in X$ such that every $\mathcal{T}$-neighborhood of $x_{0}$ is infinite. Since $X$ is open in $\bar{X}, x_{0}$ has some $\bar{X}$-neighborhood $N=\bar{X} \cap\left(G_{\alpha} \cup\left\{\beta: G_{\beta} \subset G_{\alpha}\right\}\right) \backslash F$ contained in $X$, where $F$ is finite and $x_{0} \in G \in \mathcal{T}$. Being infinite, $G_{\alpha}$ has infinitely many different infinite subsets $G_{\beta}$ in $\mathcal{T}$ (for example the sets $G_{\alpha} \backslash$ singleton); thus there are infinitely many $\beta$ 's in $N$, contradicting $N \subset X$.

That proves the "only if" part of Theorem 2; the converse implication is straightforward.

Recall that a space $X$ is "absolutely closed" if it is Hausdorff and is closed in every Hausdorff space that contains it; see, for example, [4, p. 88] for other characterizations. As in [6], $X$ is "locally absolutely closed" (here abbreviated to LAC) providing each point of $X$ has at least one absolutely closed neighborhood. ${ }^{2}$

TheOREM 3. A space $X$ is $A(F G)\left(\mathrm{T}_{2}\right)$ if and only if $X$ is Hausdorff and LAC.

That is, "absolutely locally closed" is the same as "locally absolutely closed".

The "if" implication is straightforward. For the converse, we use a construction, due to Obreanu [5], giving a special "absolute closure" $Y$ of $X$. Briefly, let $X^{\sim}$ denote the set of all free (i.e., nonconvergent) open ultrafilters on $X$, and let $Y$ be the disjoint union of $X$ and $X^{\sim} . Y$ is topologized by specifying neighborhood bases as follows. The basic neighborhoods of $x \in X$ are the sets $G \cup G^{\circ}$, where $x \in G$

\footnotetext{
${ }^{2}$ Note that points in an LAC Hausdorff space need not have arbitrarily small absolutely closed neighborhoods, because the space need not be regular.
} 
$\in \mathcal{T}$, the given topology on $X$, and where $G^{\sim}=\left\{u \in X^{\sim}: G \in u\right\}$. The basic neighborhoods of $u \in X$ are the sets $H \cup H^{r}$ where $H \in u$. It is easy to see that this produces a Hausdorff topology $\mathfrak{G}^{*}$ on $Y$. (Note that, while $Y$ agrees with the Katětov absolute closure $\kappa(X)$ as a set-as described, for instance, in [4]-its topology is different from that of $\kappa(X)$.) Clearly $(X, \mathcal{T})$ is a dense (but in general not open) subspace of $\left(Y, \mathcal{T}^{*}\right)$. It can be checked that $Y$ is absolutely closed. Now suppose $X$ is $A(\mathrm{FG})\left(\mathrm{T}_{2}\right)$; then $X$ is FG, hence open, in $Y$. Thus, fixing $x \in X$ for the moment, we have some $G \in \mathcal{T}$ such that $x \in G$ and $G^{`} \cap X^{\sim}=\varnothing$. Put $H=$ $X \backslash \mathrm{Cl}_{X}(G)$; then for each free open ultrafilter $u$ on $X, G \notin u$ and therefore (by an elementary property of open ultrafilters) $H \in u$. Thus $X^{\nu} \subset H \cup H^{\top} \subset Y \backslash G$. It follows that $\mathrm{Cl}_{Y}(G)$ is disjoint from $X^{\sim}$, and thence that $\mathrm{Cl}_{X}(G)=\mathrm{Cl}_{Y}(G)$. But it is well known (and easily verified) that the closure of an open subset of an absolutely closed space is absolutely closed. So $x$ has the absolutely closed neighborhood $\mathrm{Cl}_{X}(G)$ in $X$, proving that $X$ is LAC.

THEOREM 4. $X$ is $A(F G)$ (Tychonoff) if and only if $X$ is Hausdorff and locally compact.

"If" is a well-known special case of Theorem 3. For "only if", merely imbed $X$ in $\beta X$. The same argument proves

THEOREM $4^{\prime} . X$ is $A(F G)\left(\mathrm{T}_{4}\right)$ if and only if $X$ is locally compact and $\mathrm{T}_{4}{ }^{3}$

For completeness, we add

THEOREM 5. For a metrizable space $X$ the following are equivalent:

(1) $X$ is $A(F G)\left(\mathrm{T}_{2}\right)$,

(2) $X$ is $A(F G)$ (Metr.),

(3) $X$ is FG in every complete metric space in which it can be imbedded,

(4) $X$ is locally compact.

It is enough to prove $(3) \Rightarrow(4)$, and this is essentially what is shown in [7, Theorem 1].

REMARK. The foregoing results leave the case $A(\mathrm{FG})\left(\mathrm{T}_{3}\right)$ open; it would be interesting to have a characterization of such spaces.

2. Hereditarily absolutely FG spaces. We now consider the spaces all of whose subspaces are $A(\mathrm{FG})(\mathrm{P})$. The properties $\mathrm{P}$ arising will always be hereditary; thus any such space $X$ must certainly have the property:

(MI) Every subset of $X$ is FG in $X$.

This is equivalent to saying that every dense subset of $X$ is open, or in other words that $X$ is "maximally irresolvable" in the sense of Hewitt [3]. MI spaces are particularly interesting when they are dense-in-themselves; we abbreviate this to DMI. Hewitt shows that DMI spaces exist (satisfying the Hausdorff axiom and more), and that they are necessarily pathological. In fact, first countable spaces and

\footnotetext{
${ }^{3}$ We use " $T_{4}$ " to mean normal and $T_{1}$; similarly " $T_{3}$ " means regular $T_{1}$, and "Tychonoff" means completely regular and $T_{1}$.
} 
locally compact Hausdorff spaces, if dense-in-themselves, are always resolvable (admit disjoint dense subsets; see [3]), and thus are never MI. But MI spaces with isolated points can be quite well behaved, as Lemma 2 below shows.

Let $X$ be an arbitrary $\mathrm{T}_{1}$ space. We denote by $K(X)$ the perfect kernel of $X$ (the largest dense-in-itself subset of $X$, necessarily closed, possibly empty), and by $H(X)$ the closure of $X \backslash K(X)$. Where no ambiguity arises, we abbreviate $H(X), K(X)$ to $H, K$. Note that $H, K$ are closed sets and $H \cup K=X$.

LEMMA 1. In the notation used above, the isolated points of $H$ are dense in $H$.

This follows from the easily-verified fact that, if $D$ is the set of isolated points of $X$, then $\bar{D}=H$.

LEMMA 2. A $\mathrm{T}_{1}$ space $Y$ is $\mathrm{MI}$ and has a dense set of isolated points if, and only if, $Y^{\prime \prime}=\varnothing$.

Here $Y^{\prime}$, as usual, is the set of accumulation (= nonisolated) points of $Y$, and $Y^{\prime \prime}=\left(Y^{\prime}\right)^{\prime}$

Suppose first $D$ is a dense set of isolated points of $Y$, and $p \in Y^{\prime \prime}$. Put $E=D \cup\{p\}$. Then $\bar{E}=Y$. But $E$ is not open, for $p$ has no $Y$-neighborhood contained in $E$. This shows $Y$ is not MI, proving one implication.

Conversely, suppose $Y^{\prime \prime}=\varnothing$. To show that $Y$ is MI, it suffices to show that every dense set is open. Suppose, then, $E \subset Y$ is dense, and that $p \in E$. If $p$ is an isolated point of $Y, p$ has the neighborhood $\{p\} \in E$; so suppose $p \in Y^{\prime}$. Since $p \notin Y^{\prime \prime}, p$ has a $Y$-neighborhood $N$ containing no point of $Y^{\prime}$ other than $p$. Then $N \backslash\{p\} \subset Y \backslash Y^{\prime} \subset E$; and since also $p \in E$ we have $N \subset E$. This proves that $E$ is open, so that $Y$ is MI. And it is easy to verify that the isolated points of $Y$ are dense in $Y$.

Lemma 3. Suppose $X=Y \cup Z$, and $A \subset X$ is such that $A \cap Y$ is $F G$ in $Y$ and $A \cap Z$ is $F G$ in $Z$. Then, providing $Y$ and $Z$ are both closed, or both open, in $X$, it follows that $A$ is $F G$ in $X$.

The verification is straightforward, and is omitted.

THeOREM 6. Let $X$ be an arbitrary $T_{1}$ space. Then, in the notation of Lemma 1 , the following are equivalent:

(1) $X$ is $\mathrm{MI}$,

(2) $K$ is $\mathrm{MI}$ and $H^{\prime \prime}=\varnothing$,

(3) $X=Y \cup Z$ where $Y, Z$ are closed, $Y^{\prime \prime}=\varnothing$, and $Z$ is DMI.

(Note that in (2), $H^{\prime \prime}$ has the same meaning when the operation ' refers to the space $H$ as when it refers to $X$, because $H$ is closed in $X$. A similar remark applies to $Y^{\prime \prime}$ in (3).)

Proof. $(1) \Rightarrow(2)$. The MI property is hereditary, so $K$ and $H$ are MI. From Lemmas 1 and $2, H^{\prime \prime}=\varnothing$.

(2) $\Rightarrow(3)$. Since $K$ is dense-in-itself, this follows from Lemma 3 applied to $Y=H, Z=K$.

$(3) \Rightarrow(1)$. This follows from Lemma 3 , since $Y$ is MI by Lemma 2. 
Corollary. If $X$ is a $T_{1}$ space such that every dense-in-itself closed subspace of $X$ is resolvable-for instance, if $X$ is first countable, or locally compact and $\mathrm{T}_{2}$-then $X$ is $\mathrm{MI}$ if, and only if, $X^{\prime \prime}=\varnothing$.

For here $K=\varnothing$, so $X=H$.

REMARK. The existence of DMI Hausdorff spaces [3] shows that this corollary would not be valid without the resolvability hypothesis.

THEOREM 7. The following are equivalent, for an arbitrary Hausdorff space $X$.

(1) Every subset of $X$ is $A(F G)\left(\mathrm{T}_{2}\right)$.

(1') Every subset of $X$ is LAC.

(2) $X$ is locally compact and MI.

(3) $X$ is locally compact, and $X^{\prime \prime}=\varnothing$.

(4) Every subset of $X$ is $A(F G)$ (Tychonoff).

(5) Every subset of $X$ is locally compact.

REMARKs. (a) In particular, we have the rather surprising fact that (1) implies that $X$ must be completely regular.

(b) The equivalence of (3) and (5) is essentially the result of [2], in a somewhat simpler formulation.

Proof. The equivalence of $(1)$ and $\left(1^{\prime}\right)$ is immediate from Theorem 3; that of (2) and (3) is immediate from the Corollary to Theorem 6; and that of (4) and (5) is immediate from Theorem 4 . Since the implication $(5) \Rightarrow\left(1^{\prime}\right)$ is trivial, it will suffice to prove $(1) \Rightarrow(2)$ and $(3) \Rightarrow(5)$.

Assume (3), and let $x \in E \subset X$. Then $x$ has a compact neighborhood $N$ in $X$; and $N^{\prime \prime} \subset X^{\prime \prime}=\varnothing$. Thus, by the Corollary to Theorem 6, $N$ is MI. In particular, $N \cap E$ is FG in $N$ and is therefore locally compact. This proves that $E$ is locally compact. Thus $(3) \Rightarrow(5)$.

Finally, the implication $(1) \Rightarrow(2)$ is immediate from the following lemma.

LemMa 4. If $X$ is a Hausdorff space, then every open subset of $X$ is $A(F G)\left(\mathrm{T}_{2}\right)$ if, and only if, $X$ is locally compact.

Proof. Suppose every open subset of $X$ is $A(\mathrm{FG})\left(\mathrm{T}_{2}\right)$, and let $x \in U$, an open subset of $X$. By Theorem 3, $U$ is LAC, and therefore $x$ has a neighborhood $V$ (relative to $U$ ) that is absolutely closed. Then $V$ is a closed neighborhood of $x$ in $X$, and $V \subset U$. Thus $X$ is regular. But (again by Theorem 3) $X$ is LAC; each point has an absolutely closed neighborhood. But regular spaces that are absolutely closed are compact, so $X$ is locally compact.

The converse implication is familiar (and follows from Theorem 3).

Remark. The statement " $X$ is $\mathrm{MI}$ and $A(\mathrm{FG})\left(\mathrm{T}_{2}\right)$ " is not equivalent to the statements in Theorem 7. That is, even if every subset of the Hausdorff space $X$ is FG in $X$, and $X$ is FG in every Hausdorff space $Y$ containing $X$, it does not follow that every subset $E$ of $X$ is FG in every Hausdorff space containing $E$. For the standard example, due to Urysohn, of a noncompact absolutely closed space $X$ (see, for example, $\left[4\right.$, p. 88]) has the property that $X$ is first countable and $X^{\prime \prime}=\varnothing$; 
hence (Theorem 6, Corollary) $X$ is MI. Also $X$ is certainly LAC and therefore (Theorem 3) $A(\mathrm{FG})\left(\mathrm{T}_{2}\right)$. But it is not locally compact.

\section{REFERENCES}

1. N. Bourbaki, General topology, Part 1, Addison-Wesley, Reading, Mass., 1966.

2. H. Herrlich, V. Kannan and M. Rajagopalan, Local compactmess and simple extensions of discrete spaces, Proc. Amer. Math. Soc. 77 (1979), 421-423.

3. E. Hewitt, A problem of set-theoretic topology, Duke Math. J. 10 (1943), 309-333.

4. C.-T. Liu, Absolutely closed spaces, Trans. Amer. Math. Soc. 130 (1968), 86-104.

5. F. Obreanu, On a problem of Aleksandrov and Unysohn, Acad. Repub. Pop. Române Bul. Sti. Ser. Mat. Fiz. Chim. 2 (1950), 101-108.

6. E__ Espaces localement absolument fermés, An. Acad. Repub. Pop. Române Sect. Sti. Mat. Fiz. Chim. Ser. A 3 (1950), 375-394.

7. A. H. Stone, Absolute $F_{a}$-spaces, Proc. Amer. Math. Soc. 13 (1962), 495-499.

Department of Mathematics, University of Rochester, Rochester, New York 14627 